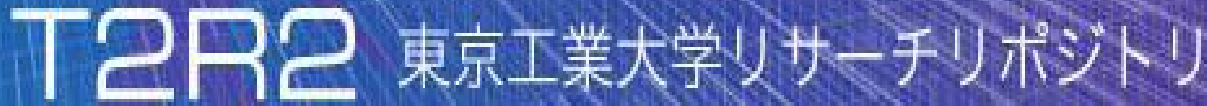

\section{Tokyo Tech Research Repository}

\section{論文 /著書情報 \\ Article /Book Information}

\begin{tabular}{|c|c|}
\hline Title & $\begin{array}{l}\text { Development of Multi-wheeled Snake-like Rescue Robots with Active } \\
\text { Elastic Trunk }\end{array}$ \\
\hline Author & Kousuke Suzuki, Atsushi Nakano, Gen Endo, Shigeo Hirose \\
\hline Journal/Book name & , , , pp. 4602-4607 \\
\hline Issue date & 2012, 10 \\
\hline DOI & http://dx.doi.org/10.1109/RO S.2012.6385757 \\
\hline URL & http://www.ieee.org/ndex.html \\
\hline Copyright & $\begin{array}{l}\text { (c) } 2012 \text { IEEE. Personal use of this material is permitted. Permission } \\
\text { from IEEE must be obtained for all other users, including } \\
\text { reprinting kepublishing this material for advertising or promotional } \\
\text { purposes, creating new collective works for resale or redistribution to } \\
\text { servers or lists, or reuse of any copyrighted components of this work in } \\
\text { other works. }\end{array}$ \\
\hline Note & $\begin{array}{l}\text { このファイルは著者 (最終) 版です。 } \\
\text { This file is author (final) version. }\end{array}$ \\
\hline
\end{tabular}




\title{
Development of Multi-wheeled Snake-like Rescue Robots with Active Elastic Trunk
}

\author{
Kousuke Suzuki $^{1}$, Atsushi Nakano ${ }^{1}$, Gen Endo ${ }^{1}$, and Shigeo Hirose ${ }^{1}$
}

\begin{abstract}
A practical robot for search and rescue operation is demanded. We believe that one of the most promising robot is a snake-like robot with a slim and active trunk. This paper proposes a new multi-wheeled snake-like robot with active elastic trunk. We choose active wheels for the propulsive device due to mechanical robustness and simplicity, and increase locomotion capability by serially arranging many active wheels along to the trunk. We also propose a new mechanism for the trunk which satisfies both active bending motion and passive compliance. The active elastic trunk consists of deformable elastic segments made of sponge and rigid (or semi-rigid) segments, where active wheel is attached, stacked in layers. The active bending motion is generated by pulling the cables passing through the trunk from the head to tail. We develop three prototypes named "Souryu-VII", "Souryu-VIII" and "SouryuIX". The mechanical design and features for each prototypes are explained and their performances are evaluated through the experiments with various measures.
\end{abstract}

\section{INTRODUCTION}

Many researchers have attempted to develop search and rescue robots in a narrow space or plowing its way through obstacles such as debris in disaster site. We believe that one of the most promising robot is a snake-like robot with a slim and active trunk because it can go into a narrow space and negotiate large obstacles by using its active trunk. The existing snake-like robots can be categorized according to their propulsive devices and trunk joints as shown in Table I. Figure 1 shows the robots which represent four major categories.

PW-AJ and PB-AJ convert the active bending motion of the trunk to the propulsive force by using a passive mechanism contacting with the ground [1][2]. The principle of its locomotion is the same as animal snake and/or earthworm. Although the principle is very interesting and worthy of investigation from a scientific point of view, the maximum thrusting force and achieved velocity are usually insufficient for practical search and rescue operations.

TABLE I

CLASSIFICATION OF SNAKE-LIKE ROBOTS

\begin{tabular}{|c|c|c|c|c|}
\hline & \multicolumn{2}{|c|}{ Joint between Segments } \\
\hline & & & Active & Passive \\
\hline \multirow{3}{*}{ Propulsive } & \multirow{3}{*}{ Active } & Wheel & AW-AJ type & AW-PJ type \\
\hline & & Crawler & AC-AJ type & - \\
\hline & & Body & - & AB-PJ type \\
\hline \multirow{2}{*}{ Mechanism } & \multirow{2}{*}{ Passive } & Wheel & PW-AJ type & - \\
\hline & & Body & PB-AJ type & - \\
\hline
\end{tabular}

*This work is supported by Grant-in-Aid for Scientific Research (A)

${ }^{1}$ K. Suzuki, A. Nakano, G. Endo and S. Hirose are with the Department of Mechano-Aerospace Engineering, Tokyo Institute of Technology, 2-12-1 Ookayama, Meguro-ku, Tokyo, 152-8552, Japan, \{gendo, hirose at mes.titech.ac.jp
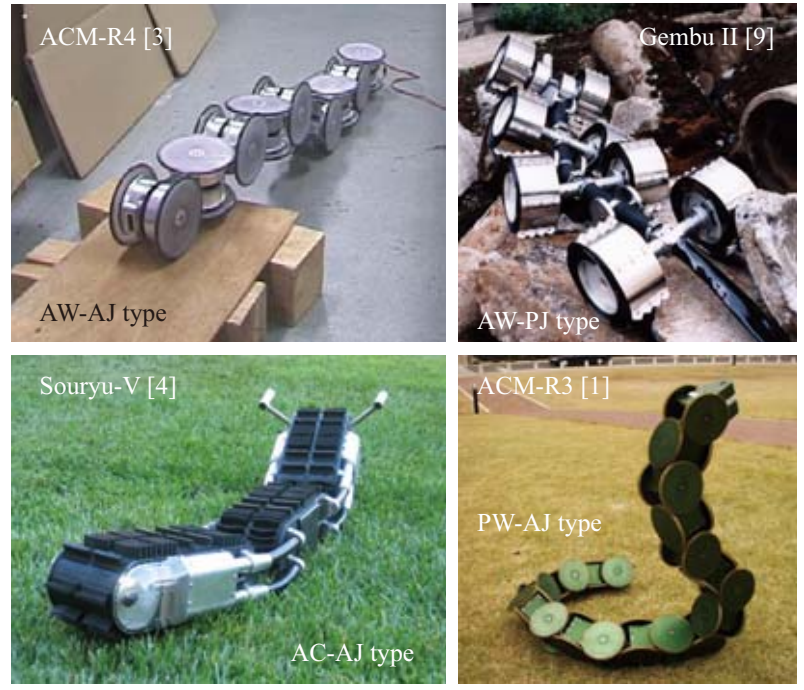

Fig. 1. Examples of previous snake-like robots

As for active propulsive mechanisms, there are three typical devices such as wheel-type [3], crawler-type [4]-[7] and cilia-type [8]. These devices can achieve larger propulsive force and velocity by utilizing the long contact area between the trunk and the ground. Although the crawler-type has the highest locomotion capability on rough terrain, it is very difficult to design water-tight and dust-proof mechanism due to many sliding parts. And crawler-type tends to be heavy and bulky. The cilia-type can be water-tight and dustproof, however, the application is still limited due to small propulsive force. While the locomotion capability of wheeltype on rough terrain is lower than the crawler-type, the wheel-type has many design advantages such as simplicity, lightweight, ease of water-tight and dust-proof.

Additionally, we can classify the snake-like robots according to joint type of the trunk. Active bending trunk can significantly improve the locomotion capability when the robot negotiates a high step or traverses a horizontal gap. However active joints might be damaged when a large external torque is suddenly applied due to unexpected drop down from piles of rubble. On the other hand, a passive compliant trunk can absorb such large external torque and protect the robot against break down. A passive compliant trunk can increase terrain adaptability on a rough environment by passively adjust each joint along the irregularity of the environment. We tested these advantages by Genbu-II [9], however, it was very difficult to control the propulsive direction due to the passive deformation of the trunk. As is obvious, it was impossible to traverse a large horizontal gap. 
Toward a practical snake-like robot for search and rescue operation, this paper proposes a new multi-wheeled snakelike robot with active elastic trunk. We choose active wheel for the propulsive device due to mechanical robustness and simplicity, and increase locomotion capability by serially arranging many active wheels along to the trunk, which can emulate a crawler. We also propose a new mechanism for the trunk which satisfies both active bending motion and passive compliance. The active elastic trunk consists of deformable elastic segments made of sponge and rigid (or semi-rigid) segments, where active wheel is attached, stacked in layers. The active bending motion is generated by pulling the wires passing through the trunk from the head to tail. The mechanism can be simple and lightweight, and it is potentially capable of both high terrain adaptability and high fault-tolerance.

In this paper, we focus on the mechanical design and experimental results to investigate feasibility of the concept and basic performance of the robot through developing and modifying three prototypes step by step.

\section{DeVElopment of SOURYu-VII}

In this section, we develop the prototype model named "Souryu-VII" which has the active elastic trunk and multiple active wheels [10]. We design Souryu-VII by considering water-tight and dust-proof mechanism.

\section{A. Mechanical Configuration}

As we previously mentioned, the wheeled robot has less terrain adaptability than the crawler robot. In order to em-

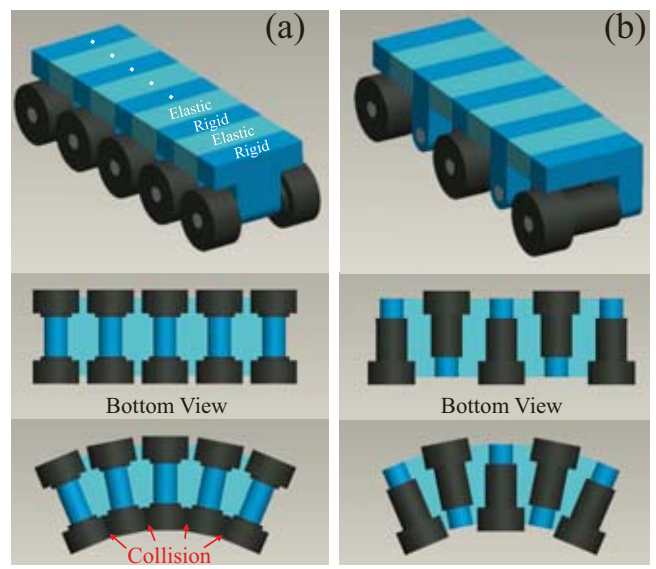

Fig. 2. Configuration of the wheels (a)conventional arrangement, (b)proposed arrangement

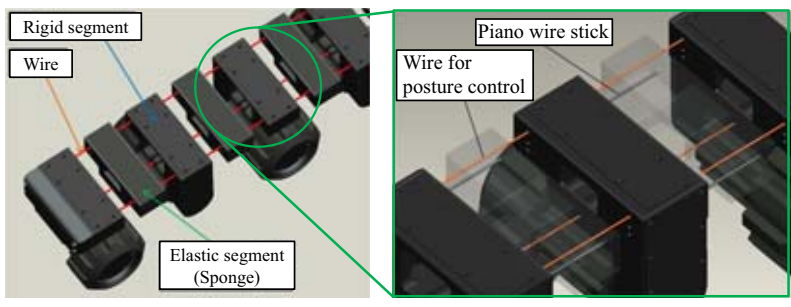

Fig. 3. Internal wire arrangement for posture change

ulate the crawler, we arrange multiple wheels along to the trunk (Fig. 2). The most straightforward implementation is to symmetrically arrange the wheels shown in Fig. 2(a). However we can not decrease the distance between the wheels because these wheels interfere with each other when the trunk bends in yaw direction. Therefore we propose to asymmetrically arrange the wheels shown in Fig. 2(b). This arrangement permits the robot to avoid wheel interference. Additionally, we can increase the traction surface to emulate a crawler belt by designing a cylindrical tire with step.

The active wheel is mounted on the rigid segment made of engineering plastic (POM), and the elastic segment is made of soft deformable rubber sponge. We design the active elastic trunk by stacking the rigid segment and elastic segment in layers (Fig. 3). We select water sealing material, ethylene propylene diene monomer (EPDM) rubber, foamed by independent bubbles for the elastic segment. There are three stainless wires inside the trunk passing through from the head to tail. One extremity of the wire is fixed to the head segment and the other extremity is connected to the external wire driving mechanism composed of a slide screw, linear guide and DC actuator (20W) through a conduit cable. The active bending motion in the yaw / pitch direction and active contracting motion in the longitudinal direction are generated by controlling the lengths of each wire. Additionally, we install two piano wires parallel to the trunk (Fig. 3(right)) in order to increase the strength against the rolling torsion and the lateral shear forces.

\section{B. Experiment}

Figure 4 shows the overview of Souryu-VII and its specification. Souryu-VII has 8 rigid segments and 7 elastic segments. There are four active wheels in the same side. Thus there are eleven actuators (eight for wheels, three for trunk) for this robot. However we synchronize four active

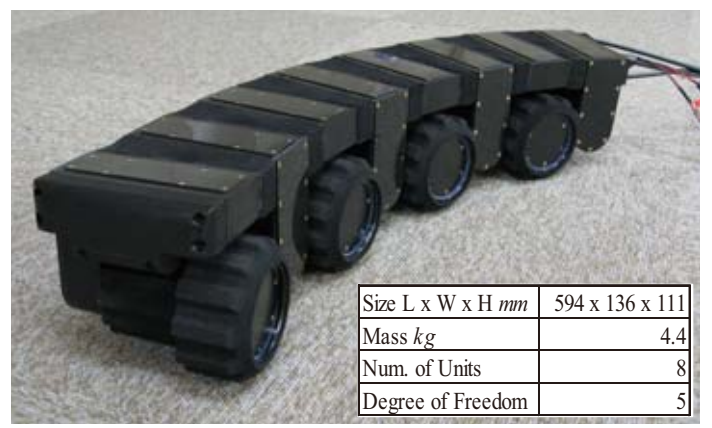

Fig. 4. Overview of the Souryu-VII and its specification

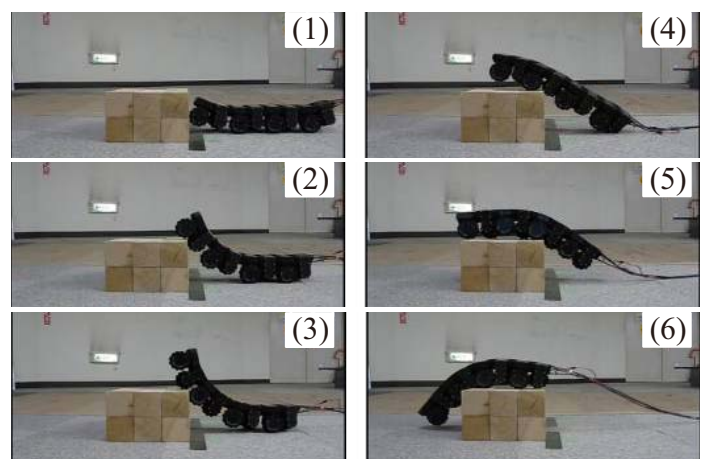

Fig. 5. Step negotiation experiment (step height $=180 \mathrm{~mm}$ ) 
wheels in the same side with the same velocity, therefore this system becomes 5 degree of freedom.

We evaluated the performance of Souryu-VII with various measures listed in Table II in section V. Figure 5 demonstrates a step climbing experiment. Souryu-VII successfully negotiated the step by lifting the front body using bending motion in the pitch direction. (The results of other experiments will be discussed in section V)

\section{Problems of Souryu-VII}

We found the following problems through the experiments.

- The bending angle is not sufficient to generate a roll over motion.

- A recovery motion is required when the robot is upsidedown.

- The friction between the wire and conduit cable is not negligible small.

- The wire is loose when an external force is applied on the trunk.

- The effectiveness of variable compliance by contraction is not remarkable.

In order to solve above problems, we develop the next prototype in the following section.

\section{Development of Souryu-VIII}

We set the following design targets for Souryu-VIII.

- Symmetric configuration with the upper and lower sides.

- Internal cable drive mechanism and cable tensioner.

- Maximization of the bending angle.

- Reduction in size and weight.

The following subsection describes the details.

\section{A. Basic Mechanical Configuration}

Figure 6 shows the basic mechanical configuration. We choose the lateral asymmetric wheel arrangement as SouryuVII. However all axis of the wheel are located in the middle of the trunk height, and the diameter of the wheel is increased in order to be symmetric configuration with the upper and lower sides.

As for the cables for the trunk bending, we arrange four cables close to the corner of the trunk indicated by the dashed lines. The upper pitching motion is generated by pulling the two upper cables and loosing the two lower cables. The right rotation around yaw axis is performed by pulling the two right cables and loosing the two left cables. We select synthetic fiber "Zylon $§$ " to reduce the weight instead of the stainless wire.

The cable driving mechanism is installed in the tail unit while the wire tensioner mechanism is in the head unit. In order to reduce the total weight of the robot, we change the material of rigid segment, where the active wheel is attached, from POM to chloroprene rubber which is harder than EPDM. This semi-rigid segment does not so much contribute to enlarge the bending angle of the trunk, however the robot becomes more impact resistant and lightweight.

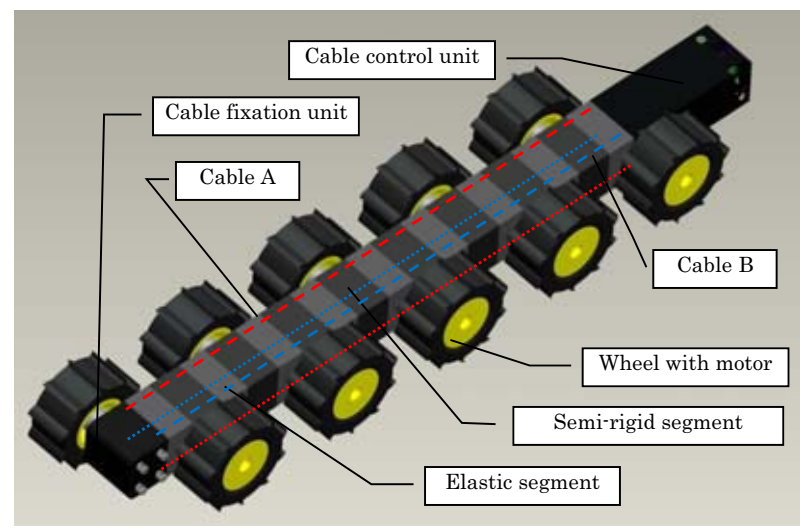

Fig. 6. Basic configuration of Souryu-VIII

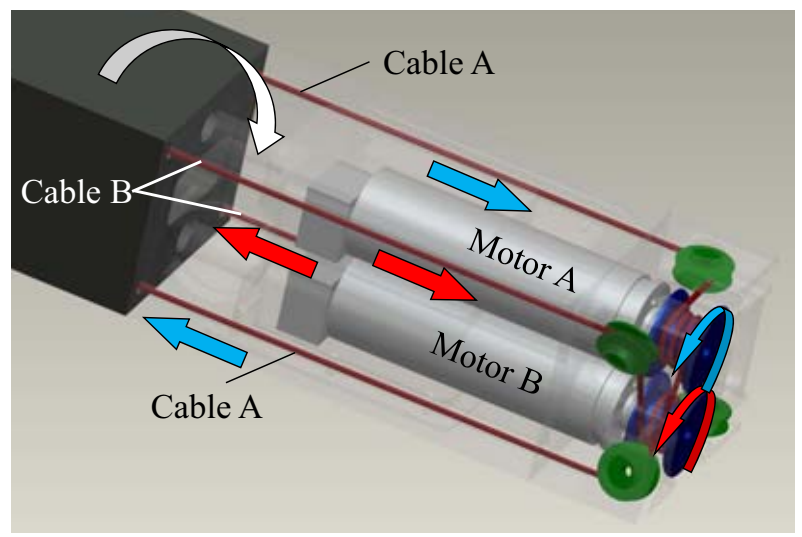

Fig. 7. Wire driving mechanism with coupled drive

\section{B. Coupled Cable Driving Mechanism and Tensioner Mech- anism}

It is not a practical design to control all four cables independently due to size and weight limitations. We propose a new mechanism to drive diagonal pair of wires at the same time (Fig. 7). The cable A passing through the right upper trunk is winded around the driving pulley of motor A via idler pulley and then the cable passes through the left lower trunk. The pathway of the cable B is symmetric to the cable A. The cables are always tensioned not to loosen by the cable tensioners installed in the head unit. This mechanism can maximize the output power of the bending motion using installed actuators because it utilizes two actuators at the same time when the trunk produces the pitching and yawing motions. In other words, this mechanism permits the robot to actuate the trunk with small lightweight actuators.

Next, we explain the cable tensioner installed in the head unit. The tensioner unit is essential because there is a trouble that the cable goes outside of the idler pulleys due to the large deformation of the trunk by a large external force. Figure 8 depicts the proposed tensioner mechanism. (Here the figure shows only one unit for the ease of explanation. There are four tensioner units in the head unit.) The extremity of the cable is fixed to the blue pulley, which is free to rotate around the green shaft, and the blue pulley is attached to the one extremity of the coil spring. Then the other extremity of the coil spring is fixed to the green shaft and the shaft can be 


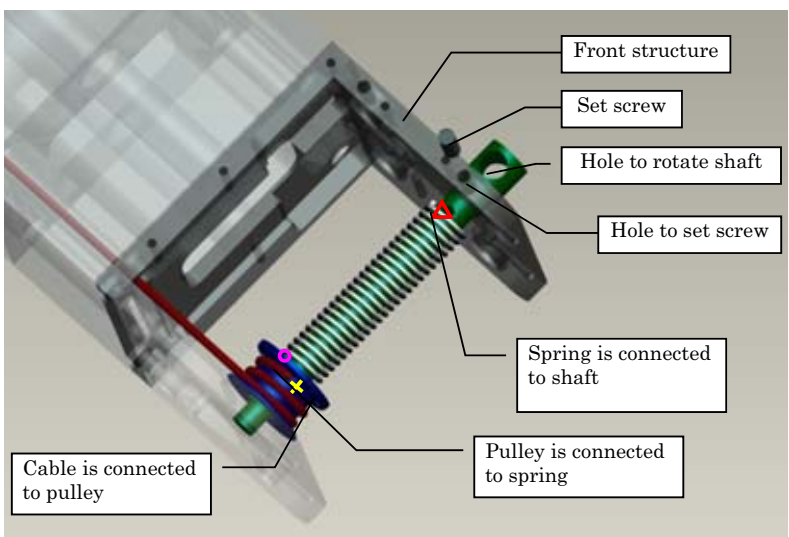

Fig. 8. Wire tensioner mechanism

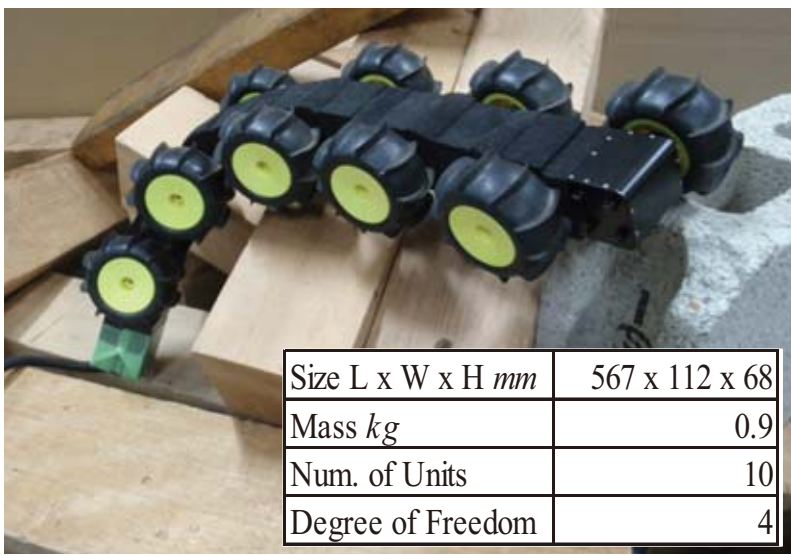

Fig. 9. Overview of the Souryu-VIII and its specification

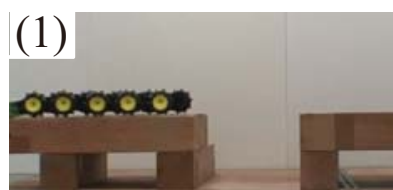

(2)

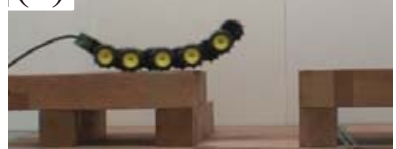

(3)

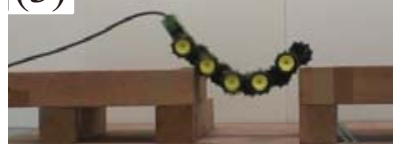

Fig. 10. Traversing a horizontal gap of $250 \mathrm{~mm}$

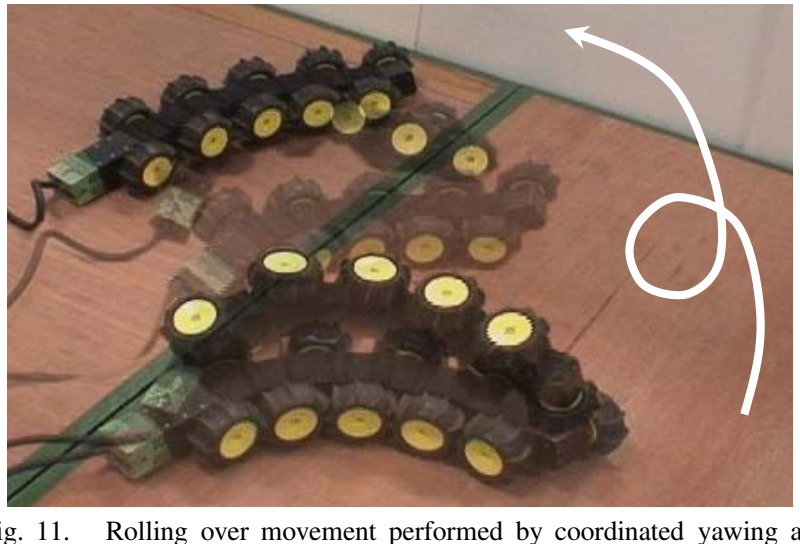

Fig. 11. Rolling over movement performed by coordinated yawing and pitching motion

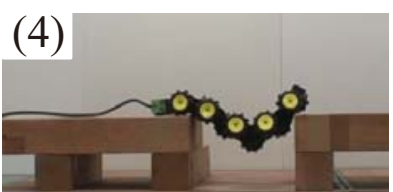

(5)

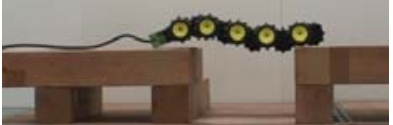

(6)

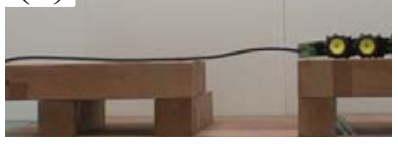

fixed to the structural part of the head unit by a set screw. The tension of the cable is adjusted by rotating the green shaft and twisting the coil spring. When the cable is pulled, the trunk is bent on condition that the deformation of the coil spring is negligible small. Thus we set the larger spring coefficient compared to the elastic trunk. When the cable is loosen, the length of the cable is adequately winded by the coil spring. This mechanism permits the four tensioners to be sufficiently compact for the head unit.

\section{Experiment}

Figure 9 shows overview of Souryu-VIII and its specification. Souryu-VIII consists of eight semi-rigid segments, nine elastic segments, the head unit and the tail unit. There are five active wheels in the same side. Thus there are twelve actuators (ten for wheels, two for trunk) for this robot. However we synchronize five active wheels in the same side with the same velocity, therefore this system becomes four degrees of freedom.

We evaluated basic performances same as Souryu-VII and Figure 10 shows the traversing a horizontal gap. At first Souryu-VIII arches the trunk backward and then lifts the trunk and finally goes across to the opposite side. Detailed experimental results are also shown in Table II.

Moreover, the rolling over motion, which was impossible for Souryu-VII, by combining pitch rotation and yaw ro- tation was achieved (Fig. 11). This recovery motion is very important when the robot rolls down from the piles of debris.

\section{Summary of Souryu-VIII}

Souryu-VIII has on-board cable driving and tensioning mechanisms and demonstrated almost the same locomotion capability compared with Souryu-VII. Moreover total weight of the robot is remarkably reduced thanks to down-sizing.

However we found that Souryu-VIII was sometimes stuck in a ditch depicted in Fig. 12 (top) during the maneuvering experiment on rough terrain. In our previous work, we did a field experiment with Souryu-IV [12] (length:1210mm) in

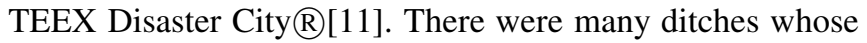
length was about $1 \mathrm{~m}$ on the piles of rubble and Souryu-IV was sometimes stuck in them. Therefore it is desirable for a practical rescue robot to be larger in size. We develop more practical prototype based on Souryu-VIII in the next section.

\section{Development of Souryu-IX}

\section{A. Basic Mechanical Configuration}

The cross-section area of a snake-like robot is design to be small in order to access a narrow space. Thus a snake-like robot tends to inherently roll down around the trunk axis. Figure 12 shows the case where the snake-like robot is stuck in the narrow ditch. It is impossible for Souryu-VII and VIII to escape from the ditch because the side surfaces of the 


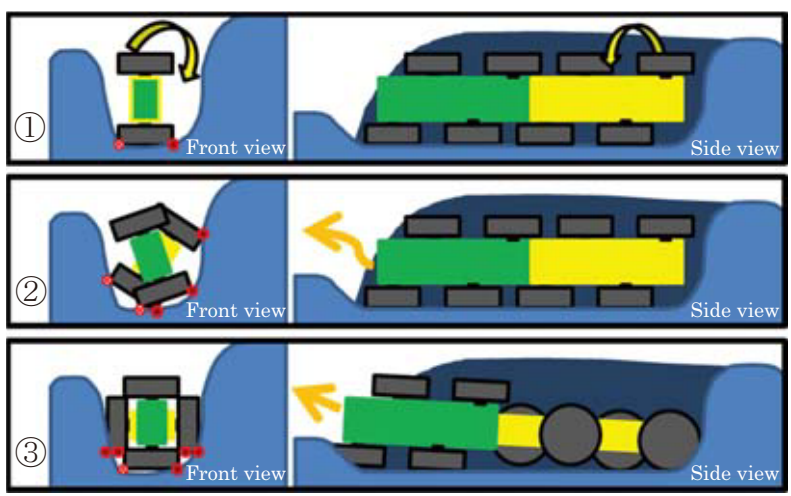

Fig. 12. Escape from a ditch by rotating trunk roll joint

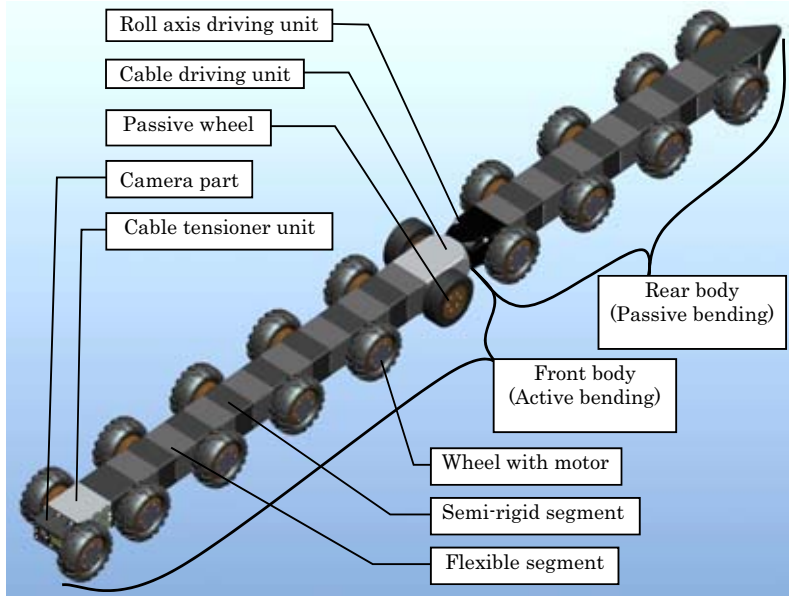

Fig. 13. Basic configuration of Souryu-IX

active wheels can not generate effective traction force. If the robot can rotate its trunk around the trunk axis (roll axis), the robot can produce sufficient thrusting force and successfully escape from the ditch by changing the contact points of the active wheels and the ground shown in Fig. 12(middle).

Fig. 13 shows the basic mechanical configuration of Souryu-IX. We design longer trunk length of $1500 \mathrm{~mm}$ and larger active wheel diameter of $100 \mathrm{~mm}$. There is an active roll joint in the middle of the trunk. In order to generate a large torque in the rolling direction, we select DC brushless actuator of $30 \mathrm{~W}$ whose reduction ratio is $1 / 1620$, achieved by a planetary gearhead, spur gears and a harmonic gear unit. There is a pipe shaft in the center of the rolling axis for the cabling.

Ideally speaking, entire trunk of the robot can generate active bending motion. However it is not practical in terms of mechanical design because we have to install two cable driving / tensioning mechanism which are relatively heavy and complicated due to the roll joint located in the middle of the trunk. Therefore we decide not to install the active bending mechanism for the rear trunk. Although the rear trunk passively follows the front trunk, we assume that the front active trunk is sufficient to negotiate rough terrain.

Additionally, we design water-tight and dust-proof mechanism for every joints, wheels and rotating parts by using oil seals and O-rings to make the robot practical. The head

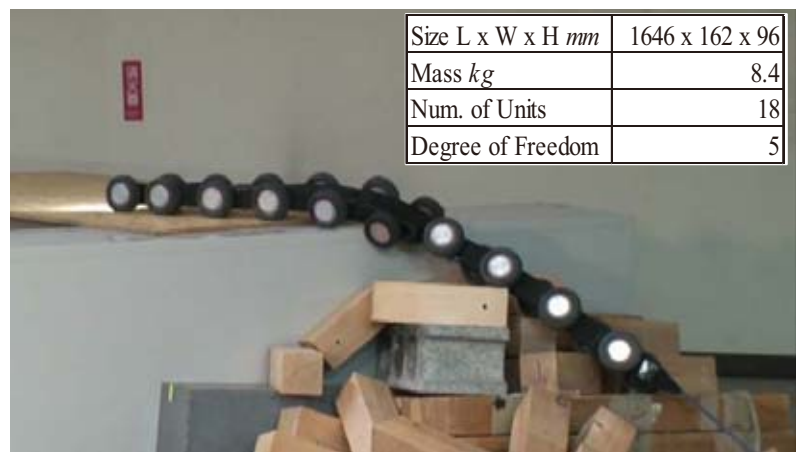

Fig. 14. Overview of Souryu-IX and its specification
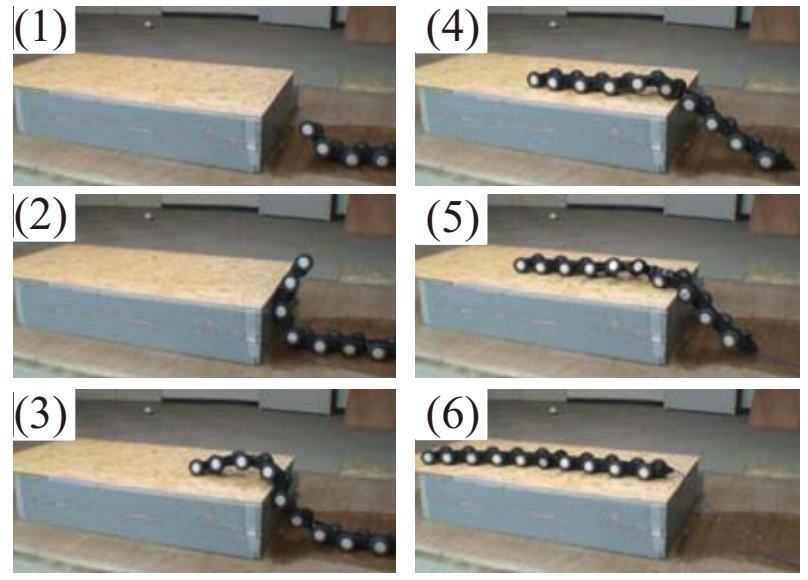

Fig. 15. Climbing experiment: step height $310 \mathrm{~mm}$

unit including LED lights and a video camera (Watec Co., Ltd.: WAT-230VIVID) is also covered for water-tight and dust-proof.

\section{B. Experiment}

Figure 14 shows overview of Souryu-IX and its specification. Souryu-IX consists of the front trunk with the active elastic trunk using eight units and the rear trunk with the passive elastic trunk using six units. The rolling mechanism and cable driving mechanism for the front trunk are located in the middle of the trunk. While eighteen active wheels are installed, two passive wheels are also used for the cable driving unit due to the space limitation. The control of the robot is basically the same as the Souryu-VIII, however, the system becomes five degrees of freedom due to the additional roll joint control.

Figure 15 shows a step climbing experiment, successfully negotiating the step by bending the front trunk. Moreover we carried out an escape experiment from a narrow ditch (Fig. 16). Despite the upper / lower object sandwiching the robot is over $30 \mathrm{~kg}$, Souryu-IX successfully escaped from the ditch by the rolling motion of the trunk to enlarge the space and change the contact points of the active wheels (Fig. 16).

\section{PERformance COMPARISON}

Table II summarizes the mobility performances of SouryuVII, VIII and XI. These three prototypes seem to have sufficient velocity of $0.3-0.4 \mathrm{~m} / \mathrm{s}$ because an operator did 

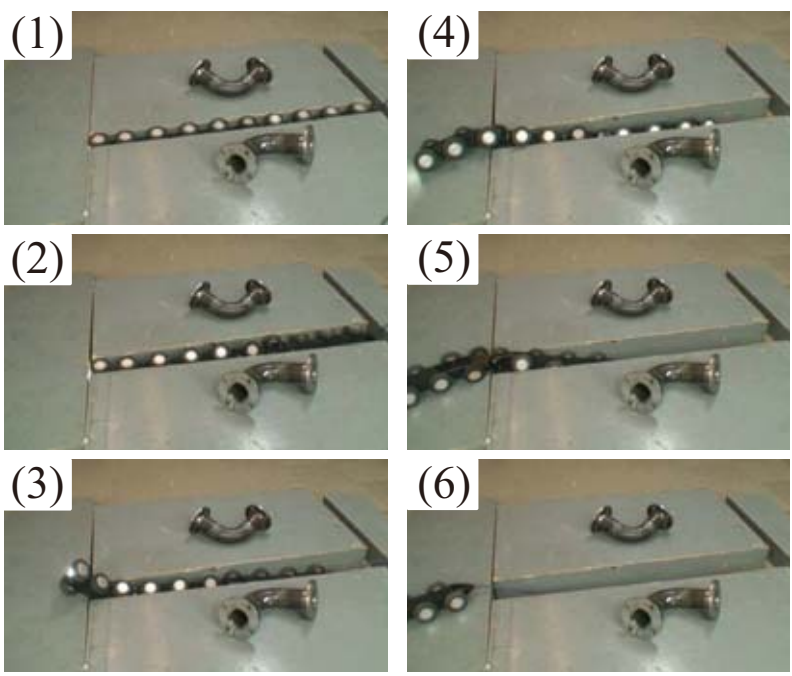

Fig. 16. Escape from a slot

TABLE II

PERFoRMANCE COMPARISON

\begin{tabular}{|l||c|c|c|}
\hline & Souryu-VII & Souryu-VIII & Souryu-IX \\
\hline Size L $\times \mathrm{W} \times \mathrm{H} \mathrm{mm}$ & $594 \times 136 \times 111$ & $567 \times 112 \times 68$ & $1646 \times 162 \times 96$ \\
\hline Mass $\mathrm{kg}$ & 4.4 & 0.9 & 8.7 \\
\hline \hline Max. Velocity $\mathrm{m} / \mathrm{s}$ & 0.315 & 0.313 & 0.398 \\
\hline Min. Turning Radius $m$ & 0.560 & 0.275 & 0.520 \\
\hline Max. Climbing Angle $\mathrm{deg}$ & 32 & 35 & 39 \\
\hline Max. Step Height $m$ & 0.180 & 0.180 & 0.310 \\
\hline Max. Horizontal Gap $m$ & 0.310 & 0.250 & 0.400 \\
\hline Pivot Turn & Good & Excellent & Good \\
\hline Rolling Over Motion & Impossible & Possible & Possible \\
\hline
\end{tabular}

not feel insufficiency during experiments. As for the minimum turning radius, Souryu-VIII is the smallest among three prototypes due to the small width. While the length of Souryu-IX is 2.8 times longer than that of Souryu-VII, the minimum turning radius of Souryu-IX is smaller than that of Souryu-VII, suggesting that the yaw bending angle of the Souryu-IX is improved.

As for pivot turn, Souryu-VIII showed very good performance on the horizontal ground and Souryu-IX is not excellent due to its long trunk. However we consider this movement is not so important because pivot turn is not possible at all on the rubble.

The performance is gradually improved for the maximum climbing angle of a slope and the rolling over motion is possible except Souryu-VII. We think that this motion is essential for the snake-like robot to recover from the rolling down.

As for the maximum step height and maximum horizontal gap, the performance of Souryu-IX is not so high considering the total length of the trunk. One reason is the heavy weight of the middle segment including the roll joint. Another reason is that only several active wheels are in contact with the ground and others can not generate traction force. This problem can be solved by optimizing the stiffness of the trunk and / or changing the material and shape of the active wheels.

\section{CONCLUSION}

In this paper, we propose a new multi-wheeled snake-like robot with active elastic trunk. We use multiple active wheels for the propulsive device mounted along to the trunk, which can emulate a crawler. We also propose a new mechanism for the trunk which satisfies both active bending motion and passive compliance. The active elastic trunk consists of deformable elastic segments and rigid (or semi-rigid) segments stacked in layers and its bending motion is generated by the cables passing through the trunk. We developed three prototypes and carried out the various experiments. Especially, Souryu-IX is water-tight and dust-proof practical design and has an ability to escape from a narrow ditch by using the roll joint located in the middle of the trunk. We plan to improve the locomotion ability of Souryu-IX on rough terrain, and water-tight and dust-proof mechanism should be evaluated by experiments in the near future.

\section{REFERENCES}

[1] M. Mori and S. Hirose, "Three-dimensional serpentine motion and lateral rolling by Active Cord Mechanism ACM-R3", Int. Conf. on Intelligent Robots and Systems, pp.829-834, 2002.

[2] S. Sugita, K. Ogami, M. Gueranieri, S. Hirose and K. Takita, "A Study on the Mechanism and Locomotion Strategy for New Snake-Like Robot Active Cord Mechanism -Slime model 1 ACM-S1", Journal of Robotics and Mechatronics, Vol.20, No.2, pp.302-309, 2008.

[3] H. Yamada and S. Hirose, "Development of Practical 3-Dimensional Active Cord Mechanism ACM-R4", Journal of Robotics and Mechatronics, Vol.18, No.3, pp.305-311, 2006.

[4] M. Arai, Y. Tanaka and S. Hirose, "Development of "Souryu-VI" and "Souryu-V:" Serially Connected Crawler Vehicles for in-Rubble Searching Operations, Journal of Field Robotics, Vol.25, No.1, pp.3165, 2008.

[5] J. Borenstein and A. Borrel, "The OminiTread OT-4 serpentine robot", Int. Conf. on Robotics and Automation, pp.1766-1767, 2008.

[6] H. Miyanaka, N. Wada, T. Kamegawa, N. Sato, S. Tsukui, H. Igarashi and F. Matsuno, "Development of an unit type robot KOHGA2 with stuck avoidance ability", Int. Conf. on Robotics and Automation, pp.3877-3882, 2007.

[7] T. Haji, T. Kinugasa, K. Yoshida, H. Amano and K. Osuka, "Experiment of maneuverability of flexible mono-tread mobile track and differential-type tracked vehicle, Industrial Robot, Vol.37, No.3, pp.263-272, 2010.

[8] K. Isaki, A. Niitsuma, M. Konyo, F. Takemura, and S. Tadokoro, "Development of an Active Flexible Cable by Ciliary Vibration Drive for Scope Camera", Int. Conf. on Intelligent Robots and Systems, pp.3946-3951, 2006.

[9] H. Kimura and S. Hirose, "Development of Genbu: Active wheel passive joint articulated mobile robot", Int. Conf. on Intelligent Robots and Systems, pp.832-828, 2002.

[10] A. Nakano and S. Hirose, "Development of Souryu-VII with active slime body and multiple wheels", Proc. of the JSME Conf. on Robotics and Mechatronics, 2P1-E19, 2009 (in Japanese).

[11] Disaster Preparedness \& Response Texas Engineering Extension Service http://www.teex.com/teex.cfm?pageid= agency\&area=usar\&templateid $=1117$

[12] M. Arai, Y. Tanaka, S. Hirose, S. Tsukui and H. Kuwahara, "Improved Driving Mechanism for Connected Crawler Vehicle Souryu-IV for in Rubble Searching Operations", Proc. SSRR, 2006 